\title{
ENERGY-CALIBRATION OF THE ATLAS HADRONIC AND ELECTROMAGNETIC LIQUID-ARGON ENDCAP CALORIMETERS
}

\author{
SVEN MENKE* \\ Max-Planck-Institut für Physik \\ (Werner-Heisenberg-Institut) \\ Föhringer Ring 6, 80805 München, Germany \\ E-mail: menke@mppmu.mpg.de
}

\begin{abstract}
In 2002 the first combined beam test of the hadronic and electromagnetic liquidargon endcap calorimeters of the ATLAS experiment took place at the SPS test beam at CERN. A total of 15 million events from electrons, muons and pions in the energy range from 6 to $200 \mathrm{GeV}$ were recorded. The entire calibration chain, from digital filter weights, over calibration constants, to clustering and energy weights, as is relevant for the energy calibration of hadronic and electromagnetic showers in ATLAS was tested and applied to the beam test data. The calibration methods and first results for the combined performance of the two calorimeters are presented.
\end{abstract}

\section{Introduction}

\subsection{The ATLAS Hadronic and Electromagnetic Endcap Calorimeters}

The ATLAS hadronic (HEC) and electromagnetic (EMEC) liquid-argon endcap calorimeters ${ }^{1-3}$ have to provide accurate measurements of jet energies and directions, missing transverse energy and particle identification in the pseudo-rapidity range $1.5 \leq|\eta| \leq 3.2$. Methods relevant for the energy calibration in ATLAS and first results of the combined performance are subject of this presentation.

\subsection{The 2002 Beam Test Setup}

$1 / 8$ wheel of the EMEC was placed in front of 3 out of 32 HEC1 modules and 2 out of 32 HEC2 half-modules in a cryostat in the $\mathrm{H} 6$ test beam area at the

*on behalf of the ATLAS Liquid Argon Collaboration 
CERN SPS. The restriction to 2 half modules of the second HEC wheel was mainly due to space constraints given by the cryostat. The 2 HEC2 halfmodules were furthermore rotated by half a $\phi$-segment around the beam axis w.r.t the nominal position in ATLAS in order to minimize the leakage of hadronic energy. In front of the first EMEC layer a presampler endcap module placed inside the cryostat allowed studies of preshower corrections with optional additional material in front of the cryostat.

The beam particles hit the calorimeters at an angle of $90^{\circ}$ in the region corresponding to $|\eta| \simeq 1.5-2.0$. Since both calorimeters have readout structures pointing in $\eta$ the observed response to electrons, pions, and muons was spread over more cells in $\eta$ than expected in ATLAS.

Scintillators for triggering and timing and 4 MWPCs with horizontal and vertical wire planes for beam position reconstruction were present further upstream in the beam line.

\section{Signal Reconstruction}

The signal reconstruction follows closely the methods deployed in previous stand-alone beam tests of the EMEC ${ }^{4}$ and the HEC ${ }^{5}$.

The output from the EMEC and from the HEC summing amplifiers were processed outside the cryostat in front-end-boards (FEB), which perform the amplification of the EMEC signals and the signal shaping for both calorimeters. A switched capacity array holds the digitized samples at a sampling rate of $40 \mathrm{MHz}$.

For each event 7 (16) samples per EMEC (HEC) channel were recorded together with the MWPC response, trigger information and the TDC measured delay between the trigger and the $40 \mathrm{MHz}$ sampling clock.

\subsection{Optimal Filtering}

The raw $\mathrm{ADC}$ samples are processed with an optimal filtering (OF) method ${ }^{6}$ using 5 samples.

For the HEC the detailed knowledge of each component in the electronics chain and the form of the input calibration pulse is used to determine the response function, which in turn is used to predict the shape for physics signals.

For the EMEC with its more complicated electronic chain a numerical method ${ }^{7}$ can be used to find the signal shape from the measured calibration response and the Fourier transformations of the ionization and the calibration current. 
The resulting predicted physics shapes together with the autocorrelation matrices from noise runs are used for the computation of the OF weights. Unlike the final situation in ATLAS with its fixed delay between trigger and sampling clock the beam test trigger comes asynchronous w.r.t. the sampling clock. Therefore the OF weights are calculated in steps of $0.5 \mathrm{~ns}$ in order to fill the $25 \mathrm{~ns}$ trigger window and parameterized by a $4^{\text {th }}$ order polynomial.

The achieved accuracy for the amplitude reconstruction following this method is better than $1.5 \%$ (2\%) for the HEC (EMEC). The OF reduces the noise of the amplitude to $64 \%(72 \%)$ of its non-filtered value in the HEC (EMEC).

DAC level scans are used to find the conversion factors from ADC counts to $\mathrm{nA}$. The linearity is found to be better than $0.5 \%$.

\section{Energy Reconstruction}

\subsection{Signal Corrections}

The non-uniformity of the E-field and sampling-fraction variations along the azimuth $(\phi)$ in the EMEC are accounted for by applying a correction (up to $2 \%$ ) derived from the signal variation found for electrons as a function of the reconstructed position in units of the cell width in the second EMEC layer. Another correction (up to 1\%) is applied to account for residual signal variations with the delay time between trigger and sampling clock. A potentially relevant variation with $\eta$ is ignored due to the narrow $\eta$-range considered in the beam test.

For the HEC a high voltage failure in 1 out of 16 LAr-gaps in the second sampling for the middle module in $\phi$ makes a correction of up to $15 \%$ in this $\phi$-region in the $2^{\text {nd }}$ layer necessary.

Following these corrections good uniformity for both calorimeters is observed.

\subsection{Clustering}

In each sampling a two-dimensional topological cluster algorithm is used to define the group of readout cells relevant for analysis. Each cluster consists of at least one cell with a signal-to-noise ratio above $4(E>4 \sigma)$. A threshold on the absolute value of the signal-to-noise ratio, $|E|>2 \sigma$, is applied to all other cells. They are included in the cluster if they share at least one edge with a cluster member cell satisfying $|E|>3 \sigma$. The 
symmetric cuts on the cell and neighbor level avoid biases due to electronics noise. Two super-clusters for the EMEC and the HEC are defined by summing all cluster signals in the EMEC and the HEC, respectively. For the HEC the signals in the $3^{\text {rd }}$ layer are multiplied by 2 in order to account for the $50 \%$ smaller sampling fraction.

\subsection{Response to Electrons}

From Monte Carlo simulations of the test beam configuration the leakage outside the EMEC was found to be very small for electrons and is neglected. Therefore the ratio of the known beam energy $(6-150 \mathrm{GeV})$ and the sum of all signals in the EMEC in $\mathrm{nA}$ defines the electromagnetic scale factor, $\alpha_{\mathrm{em}}^{\mathrm{EMEC}}=0.3855 \pm 0.004 \mathrm{MeV} / \mathrm{nA}$, where the error is statistical only. The variation with energy tests the linearity in the energy range considered and was found to be better than $0.5 \%$.

The energy resolution for electrons is studied with the super-cluster in the EMEC which contains $96-98 \%$ of the signal for high energies. Below $30 \mathrm{GeV}$ the containment falls from $96 \%$ to $90 \%$ for the lowest beam energies.

In data the resolution is found to be $\sigma_{E} / E=(0.111 \pm 0.002) / \sqrt{E / \mathrm{GeV}} \oplus$ $0 \pm 0.001$ after noise subtraction. The noise $\sigma_{\text {noise }} \simeq 0.2-0.3 \mathrm{GeV}$ varies with energy due to the non-fixed cluster size. Geant3 ${ }^{8,9}$ (Geant $4{ }^{10,11}$ ) based Monte Carlo simulations yield slightly better (worse) resolution results.

\subsection{Response to Pions}

The electromagnetic scale for the HEC is taken from the previous standalone beam test ${ }^{5}, \alpha_{\mathrm{em}}^{\mathrm{HEC}}=3.27 \pm 0.03 \mathrm{MeV} / \mathrm{nA}$, taking the modified electronics into account. Good agreement of the total visible energy in the EMEC and HEC for pions with Monte Carlo simulations based either on Geant3 or the quark-string-gluon-plasma (QGSP) model of Geant4 is observed while the Geant4 low-and-high-energy-pion-parameterization (LHEP) model deviates largely from data.

\subsection{Weighting}

The non-compensating nature of the two calorimeters makes weighting of hadronic energy deposits necessary. A cell based weighting method which was successfully used in previous experiments ${ }^{12,13}$ needs a detailed simulation on the cell level, which is not yet available for ATLAS. Therefore a more coarse weighting scheme on the super-cluster level has been applied. 
With the leakage outside the detector volume as predicted by the Monte Carlo and the known beam energy 6 weights ( 3 for the EMEC and 3 for the HEC) are fitted from the two super-cluster energies and their energy density, leading to the weighted energies $E_{\mathrm{w}}=$ $E_{\text {em }}\left(C_{1} \cdot \exp \left[-C_{2} \cdot E_{\text {em }} / V\right]+C_{3}\right)$.

The noise subtracted resolution for negative pions $\sigma_{E} / E=(0.827 \pm$ $0.003) / \sqrt{E / \mathrm{GeV}} \oplus 0 \pm 0.003$ is slightly worse than expected from Monte Carlo, which gives sampling terms around $70 \%$. For positive pions a sampling term of $(79.9 \pm 0.4) \%$ is found in data.

The ratio of the combined weighted energy of EMEC and HEC over the combined electromagnetic energy yields the effective e/ $\pi$-ratio for the endcap calorimeters ranging from 1.32 at $20 \mathrm{GeV}$ to 1.19 at $200 \mathrm{GeV}$ for negative pions and 0.05 larger values for positive pions. Geant4 based simulations predict smaller ratios by 0.02 for LHEP and 0.05 for QGSP, while Geant3 is off by -0.09 .

\section{References}

1. The ATLAS Collaboration, ATLAS liquid argon calorimeter: Technical design report, 1996, CERN-LHCC-96-41.

2. The ATLAS Collaboration, ATLAS calorimeter: Performance design report, 1997, CERN-LHCC-96-40.

3. The ATLAS Collaboration, ATLAS: Detector and physics performance technical design report. Volume 1, 1999, CERN-LHCC-99-14.

4. B. Aubert et al. (ATLAS Electromagnetic Liquid Argon Calorimeter Collab.), Nucl. Instrum. Meth. A 500 (2003) 178.

5. B. Dowler et al. (ATLAS Liquid Argon HEC Collab.), Nucl. Instrum. Meth. A 482 (2002) 94.

6. W. E. Cleland and E. G. Stern, Nucl. Instrum. Meth. A 338 (1994) 467.

7. R. Neukermans, P. Perrodo, and R. Zitoun, Understanding The Atlas Electromagnetic Barrel Pulse Shapes And The Absolute Electronic Calibration, 2001, ATL-LARG-2001-008.

8. R. Brun et al., GEANT3, 1987, CERN-DD/EE/84-1.

9. A. Kiryunin and D. Salihagić, Monte Carlo for the HEC Prototype: Software and Examples of Analysis, 1998, ATLAS HEC Note-063.

10. S. Agostinelli et al. (GEANT4 Collab.), Nucl. Instrum. Meth. A 506 (2003) 250.

11. J. P. Wellisch, http://cmsdoc.cern.ch/ ${ }^{\mathrm{hpw}} / \mathrm{GHAD} /$ HomePage.

12. I. Abt et al. (H1 Collab.), Nucl. Instrum. Meth. A 386 (1997) 348.

13. B. Andrieu et al. (H1 Calorimeter Collab.), Nucl. Instrum. Meth. A 336 (1993) 499. 